
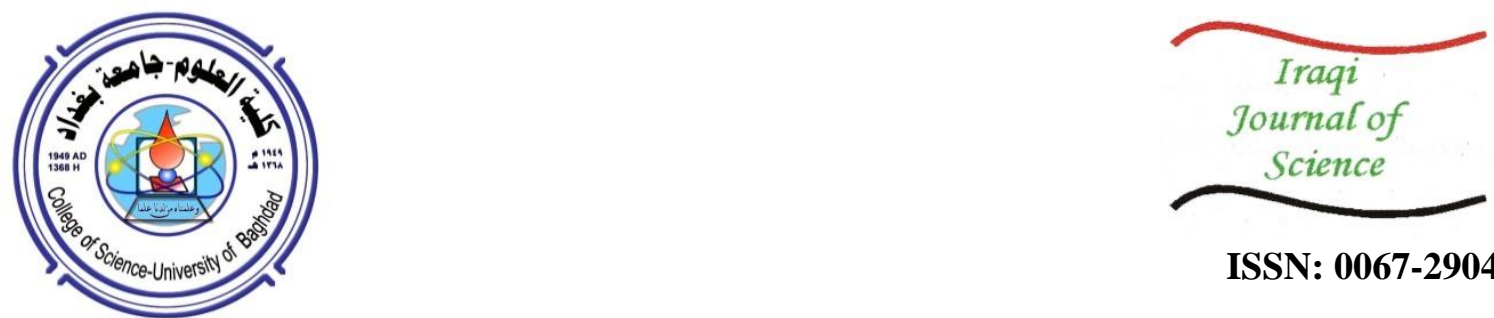

ISSN: 0067-2904

\title{
On the Dynamics of One Parameter Family of Functions $f_{k}(x)=\operatorname{kcsc}(x)$
}

\author{
Iman A. Hussain*1 ${ }^{1}$ Zeana Z. Jamil ${ }^{2}$, Nuha H. Hamada ${ }^{3}$ \\ ${ }^{I}$ Department of Mathematics and Computer Applications College of Science, Al-Nahrain University, Iraq \\ ${ }^{2}$ Department of Math., College of Science University of Baghdad, Iraq \\ ${ }^{3}$ Al Ain University, Abu Dhabi, UAE \\ Received: 22/8/2021 \\ Accepted: $1 / 11 / 2021$

\begin{abstract}
In this research, we study the dynamics of one parameter family of meromorphic functions $H=\left\{f_{k}(x)=k \csc (x): k \in \mathbb{R}\right.$ and $\left.x \in \mathbb{R}\right\}$. Furthermore, we describe the nature of fixed points of the functions in $H$, and we explain the numbers of real fixed points depending on the critical point $k$. So, we develop some necessary conditions for the convergence of the sequence $\left\{f_{k}^{n}(x)\right\}$ when $n \rightarrow \infty$.
\end{abstract}

Keywords: soft Picard iteration processes

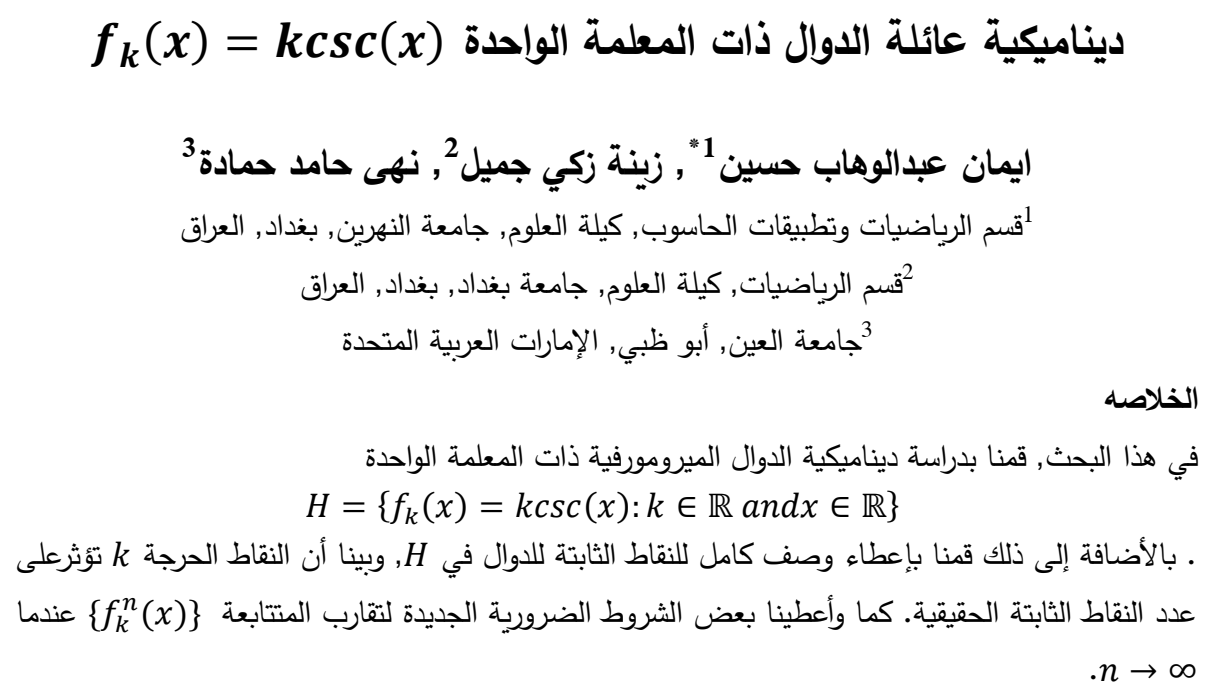

\section{1-Introduction}

Fixed point theory works as an essential tool for different branches of mathematical analysis and its applications. One of these applications is the study of real or complex dynamic function. The real dynamics of functions has been explained by Devaney [1], [2], Fadil [3] and Sajid [4],while, Akbari and Rabii [5], Magrenan and Gutierrez [6] and Radwan [7] have suggested and analyzed the real dynamics of the cubic polynomials, generalized logistic maps and one parameter family of transcendental functions. Faris [8] has discussed the dynamics of one parameter families $H=\left\{h_{k}(z)=k e^{z} /(z-1): k \in \mathbb{R}\right\}$ and $H=$ $\left\{g_{k}(z)=\frac{k \cosh (z)}{z^{2}}: k>0\right\}$ of critically and finite non-critically finite transcendental meromorphic functions respectively. For more details see [9], [10]. 
In this paper we present the real dynamics of the one parameter family $H=$ $\left\{f_{k}(x)=k \csc (x): k \in \mathbb{R}\right.$ and $\left.\left.x \in \mathbb{R}\right)\right\}$. A distinction is made between points for which $f_{k}^{n}(x)$ remains bounded as $n \rightarrow \infty$ and points for which $f_{k}^{n}(x)$ diverges. We will prove the following result.

\section{Theorem 1:}

Let $f_{k} \in H$; then there are $k_{1}, k_{2}, k_{3} \in \mathbb{R}$ such that $k_{1}<k_{2}<0<k_{3}$ such that:

1) At $k_{1}$, there exists a fixed point $x_{1}$ and $b_{1} \in \mathbb{R}$ where $f_{k_{1}}\left(b_{1}\right)=x_{1}$ satisfies :as $n \rightarrow \infty$,

i. $f_{k_{1}}^{n}(x) \rightarrow x_{1}$ when $x \in\left(b_{1}, x_{1}\right)$,

ii. $f_{k_{1}}^{n}(x) \rightarrow \infty$ when $x \in\left(\pi, b_{1}\right) \cup\left(x_{1}, 2 \pi\right)$,

iii. $f_{k_{1}}^{n}(x) \rightarrow-\infty$ when $x \in(0, \pi)$.

2) At $k_{2}$, there exist two fixed points $x_{2}$ and $r_{1} \in\left(x_{1}, 2 \pi\right)$, and $b_{2} \in \mathbb{R}$ where $f_{k_{2}}\left(b_{2}\right)=x_{2}$ satisfies : asn $\rightarrow \infty$,

i. $f_{k_{2}}^{n}(x) \rightarrow x_{2}$ when $x \in\left(b_{2}, r_{1}\right)$,

ii. $f_{k_{2}}^{n}(x) \rightarrow \infty$ when $x \in\left(\pi, b_{2}\right) \cup\left(r_{1}, 2 \pi\right)$,

iii. $f_{k_{2}}^{n}(x) \rightarrow-\infty$ when $x \in(0, \pi)$.

3) At $k_{3}$, there exist a fixed point $x_{3}$ and $b_{3} \in \mathbb{R}$ where $f_{k_{3}}\left(b_{3}\right)=x_{3}$ satisfies : as $n \rightarrow \infty$,

i. $f_{k_{3}}^{n}(x) \rightarrow x_{3}$ when $x \in\left(b_{3}, x_{3}\right)$,

ii. $f_{k_{3}}^{n}(x) \rightarrow \infty$ when $x \in\left(0, b_{3}\right) \cup\left(x_{3}, \pi\right)$,

iii. $f_{k_{3}}^{n}(x) \rightarrow-\infty$ when $x \in(\pi, 2 \pi)$.

4) At $k \in\left(k_{1}, k_{2}\right)$, there exist two fixed points $a_{1} \in\left(x_{2}, x_{1}\right), r_{2} \in\left(x_{1}, r_{1}\right)$ and $b_{4} \in \mathbb{R}$ where $f_{k}\left(b_{4}\right)=r_{2}$ satisfies : as $n \rightarrow \infty$,

i. $f_{k}^{n}(x) \rightarrow a_{2}$ when $x \in\left(b_{4}, r_{2}\right)$,

ii. $f_{k}^{n}(x) \rightarrow \infty$ when $x \in\left(\pi, b_{4}\right) \cup\left(r_{2}, 2 \pi\right)$,

iii. $f_{k}^{n}(x) \rightarrow-\infty$ when $x \in(0, \pi)$.

5) At $k \in\left(k_{2}, 0\right)$, there exists two fixed points $r_{3} \in\left(\pi, x_{2}\right), r_{4} \in\left(r_{1}, 2 \pi\right)$ and $b_{5} \in \mathbb{R}$ where $f_{k}\left(b_{5}\right)=r_{4}$ satisfy: as $n \rightarrow \infty$,

i. $f_{k}^{n}(x) \rightarrow \infty$ when $x \in\left(\pi, b_{5}\right) \cup\left(r_{4}, 2 \pi\right)$,

ii. $f_{k}^{n}(x) \rightarrow-\infty$ when $x \in(0, \pi)$,

iii. The orbit $\left\{f_{k}^{n}(x)\right\}$ is periodic or chaotic for $x \in\left(r_{3}, r_{4}\right)$.

6) At $k \in\left(0, k_{3}\right)$, there exist two fixed points $a_{2} \in\left(0, x_{3}\right), r_{5} \in\left(x_{3}, \pi\right)$ and $b_{6} \in \mathbb{R}$ where $f_{k}\left(b_{6}\right)=r_{5}$ satisfy: as $n \rightarrow \infty$,

i. $f_{k}^{n}(x) \rightarrow a_{2}$ when $x \in\left(b_{6}, r_{5}\right)$,

ii. $f_{k}^{n}(x) \rightarrow \infty$ when $x \in\left(0, b_{6}\right) \cup\left(r_{5}, \pi\right)$,

iii. $f_{k}^{n}(x) \rightarrow-\infty$ when $x \in(\pi, 2 \pi)$.

7) At $k \in\left(-\infty, k_{1}\right) \cup\left(k_{3}, \infty\right), f_{k}^{n} \rightarrow \infty$ as $n \rightarrow \infty$, for all $x \in(0,2 \pi) \backslash\{\pi\}$.

\section{2- Preliminary Results}

In this section, we describe the behavior of the fixed points of the one parameter family $H$ of transcendental meromorphic functions. Let $\phi(x): \mathbb{R} \rightarrow \mathbb{R}$ be a mapping which is defined by $\phi(x)=x \sin x$.

Now for all $f_{k} \in H$, a fixed point of $f_{k}$ must satisfy the equation $\phi(x)=k$. By solving this equation, we can find that $f_{k}$ has two fixed points $x_{1} \simeq 4.913$ and $x_{3} \simeq 2.029$. So $\phi$ has two critical values $k_{1} \simeq-4.814$ and $k_{3} \simeq 1.82$. Since $\phi$ is even and continuous then we can reduce the domain of $\phi$ to $(0, \pi)$.

The following propositions describe the number of fixed points of $f_{k}$ with respect to $k$.

Proposition 2: Let $f_{k} \in H$, then there are three cases for the number of fixed points for $f_{k}$ with respect to $k$ :

$1-f_{k}$ has no fixed point if $k<k_{1}$ or $k>k_{3}$.

2- $f_{k}$ has one fixed point at $k_{1}$ and at $k_{3}$. 
3- $f_{k}$ has two fixed points on $\left(k_{1}, 0\right)$ and on $\left(0, k_{3}\right)$.

\section{Proof: -}

let $\phi(x)=x \sin x$ and $\emptyset^{\prime}(x)=\sin x+x \cos x$ then:

$1-\phi^{\prime \prime}(x)=2 \cos x-x \sin x, \phi^{\prime \prime}\left(x_{3}\right)<0, \phi^{\prime \prime}\left(x_{1}\right)>0$ where $x_{1} \simeq 4.913$ and $x_{3} \simeq 2.029$. Thus $x_{3}$ is a maximum point and $x_{1}$ is a minimum point for $\phi(x)$ in the $(0,2 \pi)$. Then $\phi(x)=$ $k$ has no solutions for $k<k_{1}\left(k>k_{3}\right)$. So that $f_{k}$ have no fixed points in this step.

2- When $k=k_{1}\left(k=k_{3}\right)$, because of $k_{1}=\phi\left(x_{1}\right)\left(k_{3}=\phi\left(x_{3}\right)\right)$ is the minimum(maximum) value of $\phi(x)$ in $(0,2 \pi)$. Then $f_{k}$ has only one fixed point at $x=x_{1}\left(x=x_{3}\right)$.

3 - When $k \in\left(k_{1}, 0\right)$, the point $x=x_{1}$ is a minimum value in $(0,2 \pi)$. Since $\phi$ is strictly decreasing in $\left(x_{3}, x_{1}\right)$ and it is strictly increasing in $\left(x_{1}, 2 \pi\right)$, then the line $k=c$ intersects the plot of $\phi$ at exactly one point in all of the intervals $\left(x_{3}, x_{1}\right)$ and $\left(x_{1}, 2 \pi\right)$. Similarly, when $k \in$ $\left(0, k_{3}\right)$ the point $x=x_{3}$ is a maximum value in $(0,2 \pi)$. Hence $\phi$ is strictly increasing $\left(0, x_{3}\right)$ and it is strictly decreasing in $\left(x_{3}, 2 \pi\right)$, so the line $k=c$ intersects the plot of $\phi$ at exactly one point in interval $\left(0, x_{3}\right)$ and $\left(x_{3}, 2 \pi\right)$. Then $f_{k}$ has two fixed points on $\left(k_{1}, 0\right)$ and on $\left(0, k_{3}\right)$.

The purpose of the following proposition is to study the nature of fixed points of the function $f_{k}$ on $\mathbb{R}$. That is, we must study the equation $\left|f^{\prime}{ }_{k}(x)\right|=1$, since ${f^{\prime}}_{k}\left(x_{1}\right)=1=$ ${f^{\prime}}_{k}\left(x_{3}\right)$, then $x_{1}, x_{3}$ are indifferent fixed points of $f_{k}$. While the positive solution of the equation $f^{\prime}{ }_{k}(x)=\tan x-x=-1$, is $x_{2} \simeq 4.493$, hence $k_{2}=\phi\left(x_{2}\right) \simeq-4.385$.

Proposition 3:Let $f_{k} \in H$,then if:

$1-k=k_{2}$, the two fixed points of $f_{k}$ are: $x_{2}$ is indifferent, and $r_{1} \in\left(x_{2}, 2 \pi\right)$ is repelling,

2- $k \in\left(k_{1}, k_{2}\right)$, the two fixed points of $f_{k}$ are: $a_{1} \in\left(x_{1}, x_{2}\right)$ is attracting, and $r_{2} \in\left(x_{1}, r_{1}\right)$ is repelling ,

3- $k \in\left(k_{2}, 0\right)$, the two fixed points of $f_{k}$ are: $r_{3} \in\left(\pi, x_{2}\right)$ is repelling and $r_{4} \in\left(r_{1}, 2 \pi\right)$ is repelling,

4- $k \in\left(0, k_{3}\right)$,the two fixed points of $f_{k}$ are: $a_{2} \in\left(0, x_{3}\right)$ is attracting and $r_{5} \in\left(x_{3}, \pi\right)$ is repelling.

Proof: let $f_{k}(x)=k \csc x$, hence ${f^{\prime}}_{k}(x)=-k \csc x \cot x$ and the solutions of equation $k=\frac{x}{\csc x}=\phi(x)$ are the fixed points of $f_{k}$.

So $f^{\prime}{ }_{k}(x)$ at fixed point $x$ is obtained by

$$
\left|f^{\prime}{ }_{k}(x)\right|=\left|\frac{-x}{\csc x} \csc x \cot x\right|=|-x \cot x|=\frac{|x \cos x|}{|\sin x|}
$$

Now, we define the function $\mu(x)$ as follows:

$\mu(x)=|x \cos x|-|\sin x|$, it is continuous and has 3 zeros when $x=x_{1}, x_{2}$ and $x_{3}$. From the graph we can show that $\mu(x)$ is decreasing in the intervals $\left(0, x_{3}\right)$ and $\left(x_{2}, x_{1}\right)$, while it is increasing in the intervals $\left(x_{3}, x_{2}\right)$ and $\left(x_{1}, 2 \pi\right)$. So $\mu(x)$ has maximum point at $\pi$ and it has minimum point at $\mathrm{x}=-1$. From the above statements that $\mu(x)>0$;

when $x \in\left(x_{3}, x_{2}\right) \cup\left(x_{1}, 2 \pi\right), \mu(x)=0$; when $x=x_{1}, \quad x_{2}, x_{3}$ and $\mu(x)<0$ when $x \in$ $\left(0, x_{3}\right) \cup\left(x_{2}, x_{1}\right)$ see Fig $(1)$.

Thus

1- If $k=k_{2}$, the fixed point $r_{1} \in\left(x_{2}, 2 \pi\right)$ satisfies $\left|f^{\prime}{ }_{k}\left(r_{1}\right)\right|>1$, then $r_{1}$ is repelling fixed point.

2- If $k \in\left(k_{1}, k_{2}\right)$, the fixed point $a_{1} \in\left(x_{2}, x_{1}\right)$ satisfies $\left|f^{\prime}{ }_{k}\left(a_{1}\right)\right|<1$, then $a_{1}$ is an attracting fixed point. While, if the fixed point $r_{2} \in\left(x_{1}, r_{1}\right)$ satisfies $\left|f^{\prime}{ }_{k}\left(r_{2}\right)\right|>1$; then $r_{2}$ is repelling fixed point.

3- If $k \in\left(k_{2}, 0\right)$, the fixed points $r_{3} \in\left(\pi, x_{2}\right)$, and $r_{4} \in\left(r_{1}, 2 \pi\right)$ satisfy| $f^{\prime}{ }_{k}\left(r_{i}\right) \mid>1, \mathrm{i}=3,4$, then $r_{i}$ are repelling fixed points for $\mathrm{i}=3,4$. 
4- If $k \in\left(0, k_{3}\right)$, the fixed points $a_{2} \in\left(0, x_{3}\right)$ and $r_{5} \in\left(x_{3}, \pi\right)$ satisfy $\left|f^{\prime}{ }_{k}\left(a_{2}\right)\right|<1$ and $\left|f^{\prime}{ }_{k}\left(r_{5}\right)\right|>1$ respectively. Then $a_{2}$ is an attracting fixed point and $r_{5}$ is a repelling fixed point.

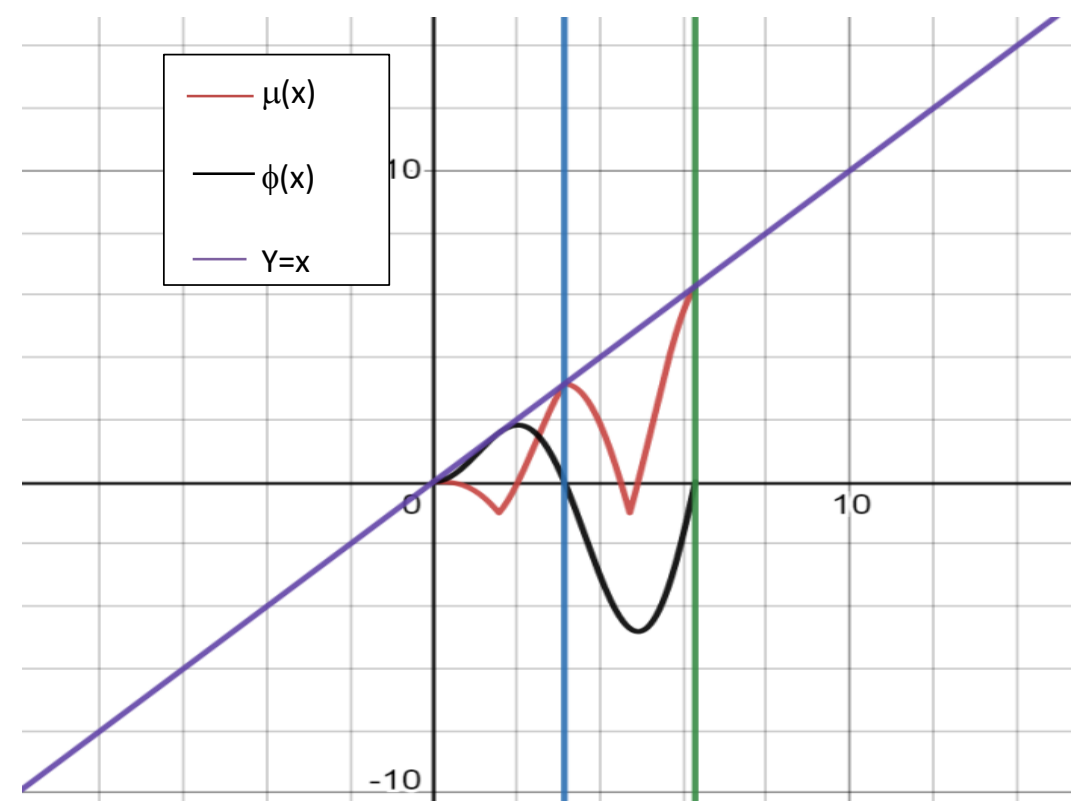

Figure 1- $\mu(x)=|x \cos x|-|\sin x|, \phi(x)=\frac{x}{\csc x}$

\section{3-The Proof of the Main result}

The proof of the main result is described as follows:

\section{Proof of the main results:}

Let $T_{k}(x)=f_{k}(x)-x$ then

1) when $\boldsymbol{k}=\boldsymbol{k}_{\mathbf{1}}, f_{k}$ has an indifferent fixed point $x_{1}$ by proposition (3). $T^{\prime}{ }_{k}\left(x_{1}\right)=0$ and $T^{\prime \prime}{ }_{k}\left(x_{1}\right)>0$, then $T_{k}$ has minimum at $x_{1}$. Because of $T_{k}\left(x_{1}\right)=0$, it follows that $T_{k}(x)>0$ for each $x$ in a neighborhood of $x_{1}$. Hence by continuity of $T_{k}$, for sufficiently small $m_{1}>0$, $T_{k}(x)>0$ in $\left(x_{1}-m_{1}, x_{1}\right) \cup\left(x_{1}, x_{1}+m_{1}\right)$. From Fig.(2) we have $T_{k}(x) \neq 0$ in $\left(\pi, x_{1}\right) \cup$ $\left(x_{1}, 2 \pi\right), T_{k}(x)>0$ for all $x \in\left(\pi, x_{1}\right) \cup\left(x_{1}, 2 \pi\right)$ and $T_{k}(x)<0$ for $x \in(0, \pi)$.

Next, we will study the dynamics of $f_{k}$ as follows:

Case(1): For $x \in\left(b_{1}, x_{1}\right) ; b_{1} \in\left(\pi, x_{1}\right) . \quad x_{1}$ is a minimum point for $T_{k} \cdot T_{k}(x)>0$ in $x \in$ $\left(\pi, x_{1}\right) \cup\left(x_{1}, 2 \pi\right)$, hence when $x \in\left(b_{1}, x_{1}\right), T^{\prime}{ }_{k}(x)<0$, so ${f^{\prime}}_{k}(x)-1<0$, then ${f^{\prime}}_{k}(x)<$ 1. Thus by the mean value theorem $\left|f_{k}(x)-f_{k}\left(x_{1}\right)\right|=f^{\prime}{ }_{k}(c)\left|x-x_{1}\right|$ such that $c \in\left(b_{1}, x_{1}\right)$. that is implies $\left|f_{k}(x)-f_{k}\left(x_{1}\right)\right|<\left|x-x_{1}\right|$ for all $x \in\left(b_{1}, x_{1}\right)$. Since $x_{1}$ is a fixed point of $f_{k}$, Thus $f_{k}^{n}(x) \rightarrow x_{1}$ as $n \rightarrow \infty$, for all $x \in\left(b_{1}, x_{1}\right)$.

Case (2):For $x \in\left(\pi, b_{1}\right) \cup\left(x_{1}, 2 \pi\right)$, then $T_{k}(x)>0$, hence $f_{k}(x)>x$, but $f_{k}\left(b_{1}\right)=x_{1}$, thus $f_{k}$ maps the interval $\left(\pi, b_{1}\right)$ into $\left(x_{1}, 2 \pi\right)$, then it is enough to prove that $f_{k}^{n}(x) \rightarrow \infty$ as $n \rightarrow \infty$ when $x \in\left(x_{1}, 2 \pi\right)$,

Since $f_{k}(x)>x$, then $\left\{f_{k}^{n}(x)\right\}$ is unbounded above and strictly increasing sequence in $x \in\left(x_{1}, 2 \pi\right)$, so $f_{k}^{n}(x) \rightarrow \infty$ as $n \rightarrow \infty$, for all $x \in\left(x_{1}, 2 \pi\right)$.

Case (3): When $x \in(0, \pi), T_{k}(x)<0$ and $f_{k}(x)<x$, therefore $f_{k}$ is strictly decreasing in this interval then $\left\{f_{k}^{n}(x)\right\}$ is decreasing sequence and it is unbounded below. So for $x \in$ $(0, \pi), f_{k}^{n}(x) \rightarrow-\infty$ as $n \rightarrow \infty$. 


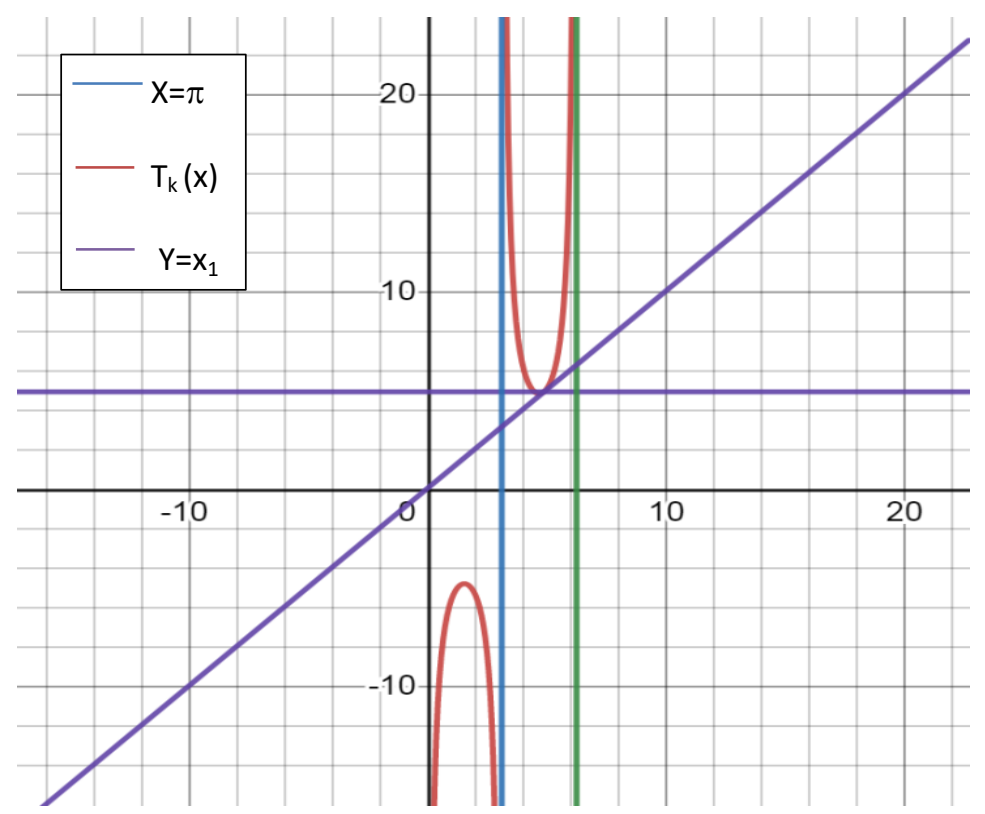

Figure 2- $T_{k}(x)=f_{k}(x)-x, k=k_{1}$

2)when $\boldsymbol{k}=\boldsymbol{k}_{\mathbf{2}}$, it is clear from Fig. (3) $T_{k}(x)>0$, for all $x \in\left(\pi, x_{2}\right) \cup\left(r_{1}, 2 \pi\right)$ and $T_{k}(x)<0$ for all $x \in\left(x_{2}, r_{1}\right) \cup(0, \pi)$.

Now, we can describe the dynamic of $f_{k}$.

Case (1): For $x \in\left(b_{2}, r_{1}\right)$, we will show that $f_{k}^{n}(x) \rightarrow x_{2}$ since $T_{k}(x)<0$ for $x \in\left(x_{2}, r_{1}\right)$ then $f_{k}(x)<x$. Since $f_{k}$ is decreasing and by continuity forward iteration process we get $x>f_{k}(x)>f_{k}^{2}(x)>\cdots>f_{k}^{n}(x)>x_{2}$.

Therefore, the sequence $\left\{f_{k}^{n}(x)\right\}$ is decreasing and bounded below by $x_{2}$. So $f_{k}^{n}(x) \rightarrow x_{2}$ as $n \rightarrow \infty$ for $x \in\left(x_{2}, r_{1}\right)$.

Further since $f_{k}\left(b_{2}\right)=r_{1}$, and it is decreasing in $\left(b_{2}, r_{1}\right), f_{k}$ maps the interval $\left(b_{2}, r_{1}\right)$ into $\left(x_{2}, r_{1}\right)$.It follows that byusing the previous arguments, $f_{k}^{n}(x) \rightarrow x_{2}$ as $n \rightarrow \infty$ for $x \in\left(b_{2}, r_{1}\right)$. Case (2): For $x \in\left(r_{1}, 2 \pi\right), f_{k}(x)>x$. Moreover $f_{k}$ is strictly increasing in this interval, then

$$
0<x<f_{k}(x)<f_{k}^{2}(x)<\cdots<f_{k}^{n}(x)<\cdots
$$

Thus, the sequence $\left\{f_{k}^{n}(x)\right\}$ is increasing and it is unbounded above. Hence $f_{k}^{n}(x) \rightarrow-\infty$ as $n \rightarrow \infty$ for $x \in\left(r_{1}, 2 \pi\right)$.

Now, for $x \in\left(\pi, b_{2}\right) ; b_{2} \in\left(\pi, x_{2}\right)$, we have $f_{k}(x)>x . f_{k}\left(b_{2}\right)=r_{1}$. Then $f_{k}$ maps the interval $\left(\pi, b_{2}\right)$ into $\left(r_{1}, 2 \pi\right)$. Thus $f_{k}^{n}(x) \rightarrow-\infty$ as $n \rightarrow \infty$ for $x \in\left(\pi, b_{2}\right) \cup\left(r_{1}, 2 \pi\right)$.

Case (3): For $x \in(0, \pi)$ from Fig.(3), we have $T_{k}(x)<0$ then $f_{k}(x)<x$ so that $f_{k}$ is strictly decreasing in this interval, and hence

$$
x>f_{k}(x)>f_{k}^{2}(x)>f_{k}^{3}(x)>\cdots>f_{k}^{n}(x)>\cdots
$$

Thus $\left\{f_{k}^{n}(x)\right\}$ is decreasing sequence, which is unbounded below. Therefore, for $x \in(0, \pi)$ we have $f_{k}^{n}(x) \rightarrow-\infty$ as $n \rightarrow \infty$. 


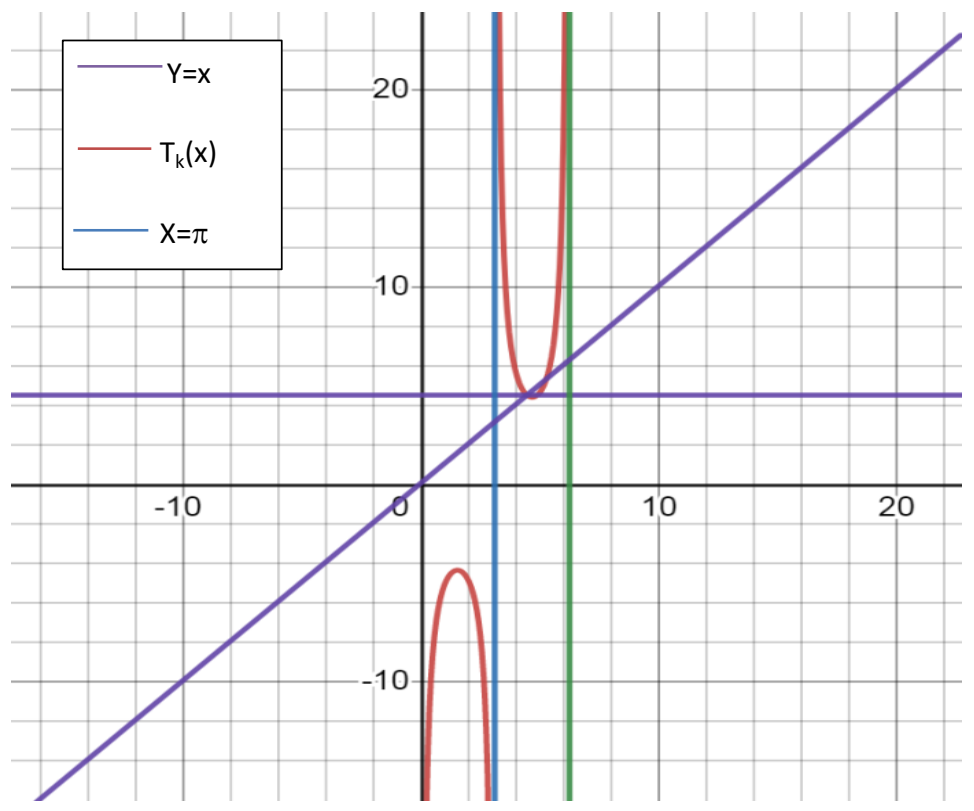

Figure 3- $T_{k}(x)=f_{k}(x)-x, k=k_{2}$

3)when $\boldsymbol{k}=\boldsymbol{k}_{\mathbf{3}}, f_{k}$ has an indifferent fixed point $x_{3}$ by proposition (3). $T^{\prime}{ }_{k}\left(x_{3}\right)=0$, and $T^{\prime \prime}{ }_{k}\left(x_{3}\right)>0$, then $T_{k}$ has minimum at $x_{3}$. Because of $T^{\prime}{ }_{k}\left(x_{3}\right)=0$, it follows that $T_{k}(x)>0$ for each $x$ in a neighborhood of $x_{3}$. Hence by continuity of $T_{k}$, for sufficiently small $m_{1}>0$, $T_{k}(x)>0$ in $x \in\left(x_{3}-m_{1}, x_{3}\right) \cup\left(x_{3}, x_{3}+m_{1}\right)$. From Fig.(4) we have $T_{k}(x) \neq 0$ in $x \in\left(0, x_{3}\right) \cup\left(x_{3}, \pi\right)$, so $T_{k}(x)>0$ for all $x \in\left(0, x_{3}\right) \cup\left(x_{3}, \pi\right)$, and $T_{k}(x)<0$ for $x \in$ $(\pi, 2 \pi)$.

Next, we will study the dynamics of $f_{k}$ as follow:

Case(1):For $x \in\left(b_{3}, x_{3}\right) ; b_{3} \in\left(0, x_{3}\right), x_{3}$ is a minimum point for $T_{k} . T_{k}(x)>0$ in $\left(0, x_{3}\right) \cup$ $\left(x_{3}, \pi\right)$, hence when $x \in\left(b_{3}, x_{3}\right), T^{\prime}{ }_{k}(x)<0$, so $T_{k}(x)<0$, then ${f^{\prime}}_{k}(x)<1$. For $x>x_{3}$, ${f^{\prime}}^{\prime}(x)>1$. Thus by the mean value theorem $\left|f_{k}(x)-f_{k}\left(x_{1}\right)\right|=f^{\prime}{ }_{k}(c)\left|x-x_{1}\right|$ such that $c \in\left(b_{3}, x_{3}\right)$. Since $x_{3}$ is a fixed point of $f_{k}$, that is implies $\left|f_{k}(x)-f_{k}\left(x_{3}\right)\right|<\left|x-x_{3}\right|$ for all $x \in\left(b_{3}, x_{3}\right)$. Thus $f_{k}^{n}(x) \rightarrow x_{3}$ as $n \rightarrow \infty$, for all $x \in\left(b_{3}, x_{3}\right)$.

Case (2):for $x \in\left(0, b_{3}\right) \cup\left(x_{3}, \pi\right)$ then $T_{k}(x)>0$ for all $x \in\left(x_{3}, \pi\right)$, hence $f_{k}(x)>x$, since $f_{k}$ is strictly increasing in this interval, and

$$
0<x<f_{k}(x)<f_{k}^{2}(x)<\cdots<f_{k}^{n}(x)<\text {.. }
$$

Then $\left\{f_{k}^{n}(x)\right\}$ is increasing sequence which it is unbounded, above so $f_{k}^{n}(x) \rightarrow \infty$ as $n \rightarrow \infty$, for all $x \in\left(x_{3}, \pi\right)$. Since $f_{k}\left(b_{3}\right)=x_{3}$ and $f_{k}$ maps the interval $\left(0, b_{3}\right)$ into $\left(x_{3}, \pi\right)$ hence we can use the same arguments to prove $f_{k}^{n}(x) \rightarrow \infty$ as $n \rightarrow \infty$ when $x \in\left(0, b_{3}\right)$.

Case (3): when $x \in(\pi, 2 \pi), T_{k}(x)<0$ then $f_{k}(x)<x$, therefore $f_{k}$ is strictly decreasing in this interval and $x_{3}>f_{k}(x)>f_{k}^{2}(x)>\cdots>f_{k}^{n}(x)>\cdots$.

Then $\left\{f_{k}^{n}(x)\right\}$ is decreasing sequence and it is unbounded below. So for $x \in(\pi, 2 \pi) f_{k}^{n}(x) \rightarrow$ $-\infty$ as $n \rightarrow \infty$. 


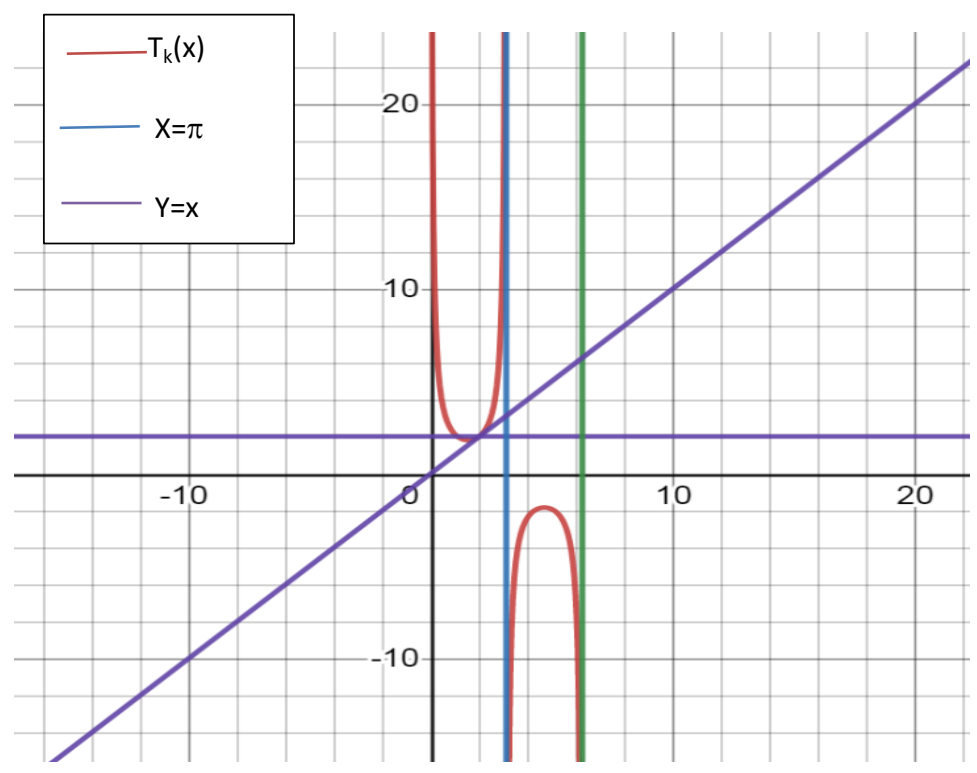

Figure $4-T_{k}(x)=f_{k}(x)-x, k=k_{3}$

4)when $\boldsymbol{k} \in\left(\boldsymbol{k}_{1}, \boldsymbol{k}_{2}\right)$, by proposition (3) $f_{k}$ has an attracting fixed point $a_{1} \in\left(x_{2}, x_{3}\right)$ and repelling fixed point $r_{2} \in\left(x_{1}, r_{1}\right)$. From Fig. $(5) T_{k}(x) \neq 0$ in $\left(\pi, r_{2}\right) \cup\left(r_{2}, 2 \pi\right), T_{k}(x)>0$ for all $x \in\left(\pi, a_{1}\right) \cup\left(r_{2}, 2 \pi\right)$ and $T_{k}(x)<0$ for $x \in(0, \pi) \cup\left(a_{2}, r_{2}\right)$.

To describe the dynamics of $f_{k}$, we have three cases:-

Case(1) when $x \in\left(b_{4}, r_{2}\right) . f_{k}(x)<x$ for all $x \in\left(a_{1}, r_{2}\right)$ and it is decreasing, so $x>f_{k}(x)>f_{k}^{2}(x)>\cdots>f_{k}^{n}(x)>a_{1}$.

Hence the sequence $\left\{f_{k}^{n}(x)\right\}$ is decreasing and bounded below by $a_{1}$, and there is no fixed point larger than $a_{1}$. Therefore $f_{k}^{n}(x) \rightarrow a_{1}$ as $n \rightarrow \infty$ for all $x \in\left(b_{4}, r_{2}\right)$.

Case (2):-For $x \in\left(\pi, b_{4}\right) \cup\left(r_{2}, 2 \pi\right), f_{k}(x)>x$ for all $x \in\left(r_{2}, 2 \pi\right)$. Since $f_{k}$ is increasing and by continuing forward iteration process, it follows $0<x<f_{k}(x)<f_{k}^{2}(x)<\cdots<f_{k}^{n}(x)<\cdots$

Hence, the sequence $\left\{f_{k}^{n}(x)\right\}$ is increasing and there is no fixed point larger than $r_{2}$, the orbit must go to $\infty$ as $n \rightarrow \infty$. Then $f_{k}^{n}(x) \rightarrow \infty$ as $n \rightarrow \infty$ for all $x \in\left(r_{2}, 2 \pi\right) . f_{k}\left(b_{4}\right)=r_{2}, f_{k}$ maps the interval $\left(\pi, b_{4}\right)$ into $\left(r_{2}, 2 \pi\right)$. Then by using the above arguments $f_{k}^{n}(x) \rightarrow \infty$ as $n \rightarrow \infty$ for all $x \in\left(\pi, b_{4}\right)$.

Case (3): for $x \in(0, \pi), T_{k}(x)<0$, hence $f_{k}(x)<x$, therefore $f_{k}$ is strictly decreasing in this interval and

$x>f_{k}(x)>f_{k}^{2}(x)>\cdots>f_{k}^{n}(x)>\cdots$.

Then $\left\{f_{k}^{n}(x)\right\}$ is decreasing sequence and it is unbounded below. So for $x \in(0, \pi) f_{k}^{n}(x) \rightarrow$ $-\infty$ as $n \rightarrow \infty$. 


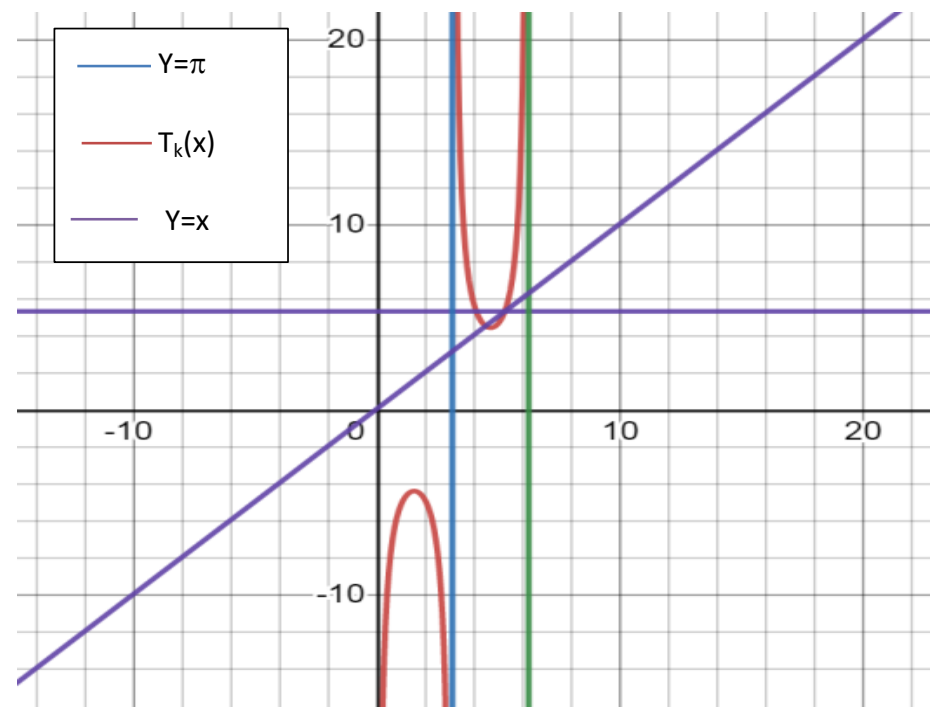

Figure 5- $T_{k}(x)=f_{k}(x)-x, \mathrm{k} \in\left(\mathrm{k}_{1}, \mathrm{k}_{2}\right)$

5)when $\boldsymbol{k} \in\left(\boldsymbol{k}_{2}, \mathbf{0}\right), f_{k}$ has two fixed points $r_{3} \in\left(\pi, x_{2}\right)$ and $r_{4} \in\left(r_{1}, 2 \pi\right)$ which are repelling by proposition (3). From Fig(6) we have $T_{k}(x)>0$ for all $x \in\left(\pi, b_{5}\right) \cup\left(r_{4}, 2 \pi\right)$ and $T_{k}(x)<0$ for $x \in(0, \pi) \cup\left(r_{3}, r_{4}\right)$.

To describe the dynamics of $f_{k}$, we have three cases:-

Case (1):- for $x \in\left(r_{4}, 2 \pi\right), f_{k}(x)>x$. Hence $f_{k}$ is strictly increasing in $\left(r_{4}, 2 \pi\right)$, then $0<r_{4}<x<f_{k}(x)<f_{k}^{2}(x)<\cdots<f_{k}^{n}(x)<\cdots$.

So the sequence $\left\{f_{k}^{n}(x)\right.$ \}is increasing sequence which is unbounded above. So $f_{k}^{n}(x) \rightarrow \infty$ as $n \rightarrow \infty$ for $x \in\left(r_{4}, 2 \pi\right)$. Then $f_{k}^{n}(x) \rightarrow \infty$ as $n \rightarrow \infty$ for all $x \in\left(\pi, r_{4}\right)$. Because $f_{k}\left(b_{6}\right)=$ $r_{5}$, and $f_{k}$ maps the interval $\left(\pi, r_{4}\right)$ into $\left(r_{4}, 2 \pi\right)$. By using the above arguments $f_{k}^{n}(x) \rightarrow \infty$ as $n \rightarrow \infty$ for all $x \in\left(\pi, r_{4}\right)$.

Case (2):- for $x \in\left(r_{3}, r_{4}\right)$ the system of dynamics of $f_{k}$ has no point attractors. Thus dynamical system will move indefinitely, and the orbit $\left\{f_{k}^{n}(x)\right\}$ will be periodic or chaotic in these intervals.

Case (3):-when $\in(0, \pi), T_{k}(x)<0$ then $f_{k}(x)<x$. Therefore $f_{k}$ is strictly decreasing in this interval and

$$
x_{1}>f_{k}(x)>f_{k}^{2}(x)>\cdots>f_{k}^{n}(x)>\cdots
$$

Then $\left\{f_{k}^{n}(x)\right\}$ is decreasing sequence and it is unbounded below. So for $x \in(0, \pi), f_{k}^{n}(x) \rightarrow$ $-\infty$ as $n \rightarrow \infty$. 


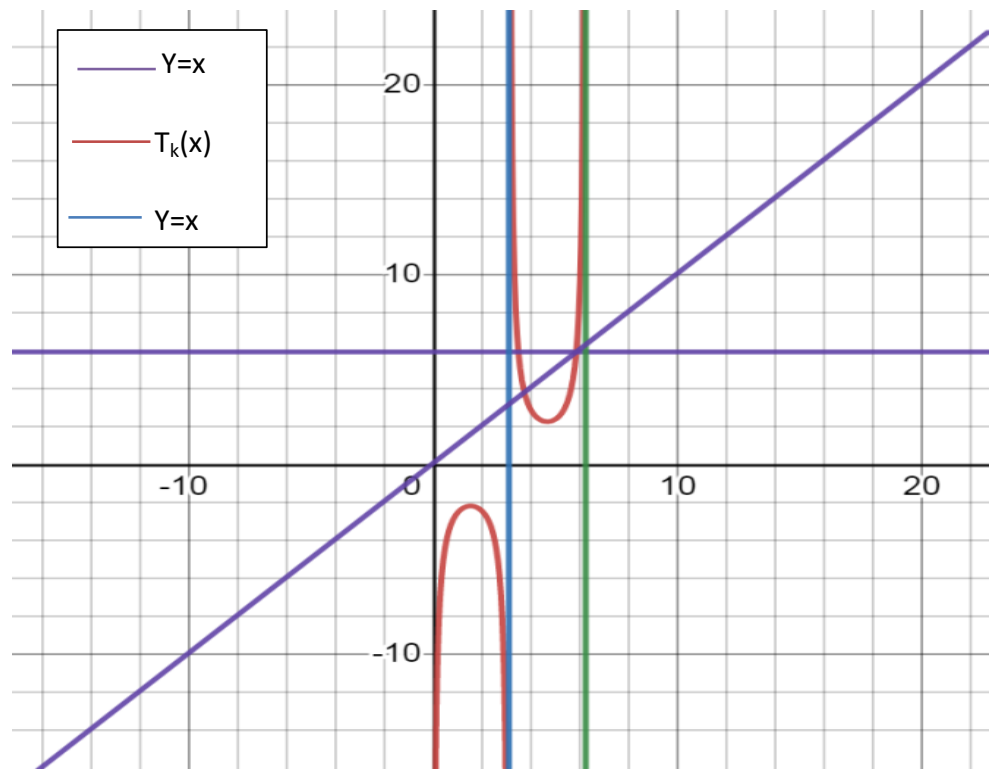

Figure 6- $T_{k}(x)=f_{k}(x)-x, \mathrm{k} \in\left(\mathrm{k}_{2}, 0\right)$

6) when $\boldsymbol{k} \in\left(\mathbf{0}, \boldsymbol{k}_{\mathbf{3}}\right)$, so $f_{k}$ has an attracting fixed point $a_{2} \in\left(0, x_{3}\right)$ and repelling fixed point $r_{5} \in\left(x_{3}, \pi\right)$ by proposition (3). From Fig. (7) we have $T_{k}(x)>0$ for all $x \in\left(0, b_{6}\right) \cup\left(r_{5}, \pi\right)$ and $T_{k}(x)<0$ for $x \in(\pi, 2 \pi) \cup\left(a_{2}, r_{5}\right)$.

To describe the dynamics of $f_{k}$, we have three cases:-

Case(1) when $x \in\left(b_{6}, r_{5}\right)$, then $f_{k}(x)<x$ and it is decreasing, so

$$
x>f_{k}(x)>f_{k}^{2}(x)>\cdots>f_{k}^{n}(x)>\cdots>a_{2}
$$

Hence the sequence $\left\{f_{k}^{n}(x)\right\}$ is decreasing and bounded below by $a_{2}$, and there is no fixed point larger than $a_{2}$. Therefore $f_{k}^{n}(x) \rightarrow a_{2}$ as $n \rightarrow \infty$ for all $x \in\left(b_{6}, r_{5}\right)$.

Case (2):- for $x \in\left(0, b_{6}\right) \cup\left(r_{5}, \pi\right), f_{k}(x)>x$ for all $x \in\left(r_{5}, \pi\right)$. Since $f_{k}$ is increasing and by continuing forward iteration process, it follows that

$$
0<x<f_{k}(x)<f_{k}^{2}(x)<\cdots<f_{k}^{n}(x)<\cdots
$$

Hence, the sequence $\left\{f_{k}^{n}(x)\right\}$ is increasing and there is no fixed point larger than $r_{5}$, the orbit must go to $\infty$ as $n \rightarrow \infty$. Then $f_{k}^{n}(x) \rightarrow \infty$ as $n \rightarrow \infty$ for all $x \in\left(r_{5}, \pi\right) \cdot f_{k}\left(b_{6}\right)=r_{5}$ and $f_{k}$ maps the interval $\left(r_{5}, \pi\right) \operatorname{into}\left(0, b_{6}\right)$. So by using the above arguments we can getting $f_{k}^{n}(x) \rightarrow \infty$ as $n \rightarrow \infty$ for all $x \in\left(0, b_{6}\right)$.

Case (3): for $x \in(\pi, 2 \pi), T_{k}(x)<0$ then $f_{k}(x)<x$, therefore $f_{k}$ is strictly decreasing in this interval and

$$
x>f_{k}(x)>f_{k}^{2}(x)>\cdots>f_{k}^{n}(x)>\cdots .
$$

Then $\left\{f_{k}^{n}(x)\right\}$ is decreasing sequence and it is unbounded below. So for all $x \in(\pi, 2 \pi), f_{k}^{n}(x) \rightarrow-\infty$ as $n \rightarrow \infty$. 


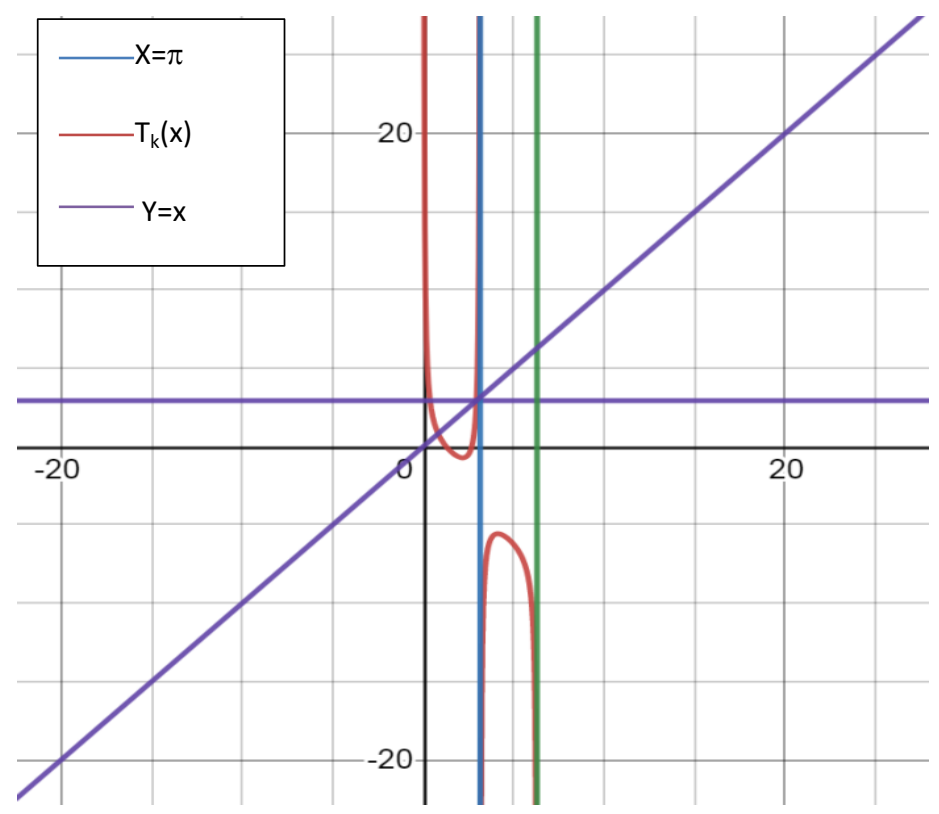

Figure 7- $T_{k}(x)=f_{k}(x)-x, \mathrm{k} \in\left(0, \mathrm{k}_{3}\right)$

7)when $\mathbf{k}<k_{\mathbf{1}}\left(\boldsymbol{k}>\boldsymbol{k}_{\mathbf{3}}\right), f_{k}$ has no fixed point by proposition (2). Since $f_{k}$ is continues and differential for $x \in(0,2 \pi)$ then $T_{k}$ is continuous and differentiable. From Fig. (8) the sequence $\left\{f_{k}^{n}(x)\right\}$ is increasing and it is unbounded below for all $x \in(0,2 \pi)$. Hence $f_{k}^{n}(x) \rightarrow \infty$ as $n \rightarrow-\infty$ for all $x \in(0,2 \pi)$. When $k>k_{3}$ the proof is similar to the previous arguments.

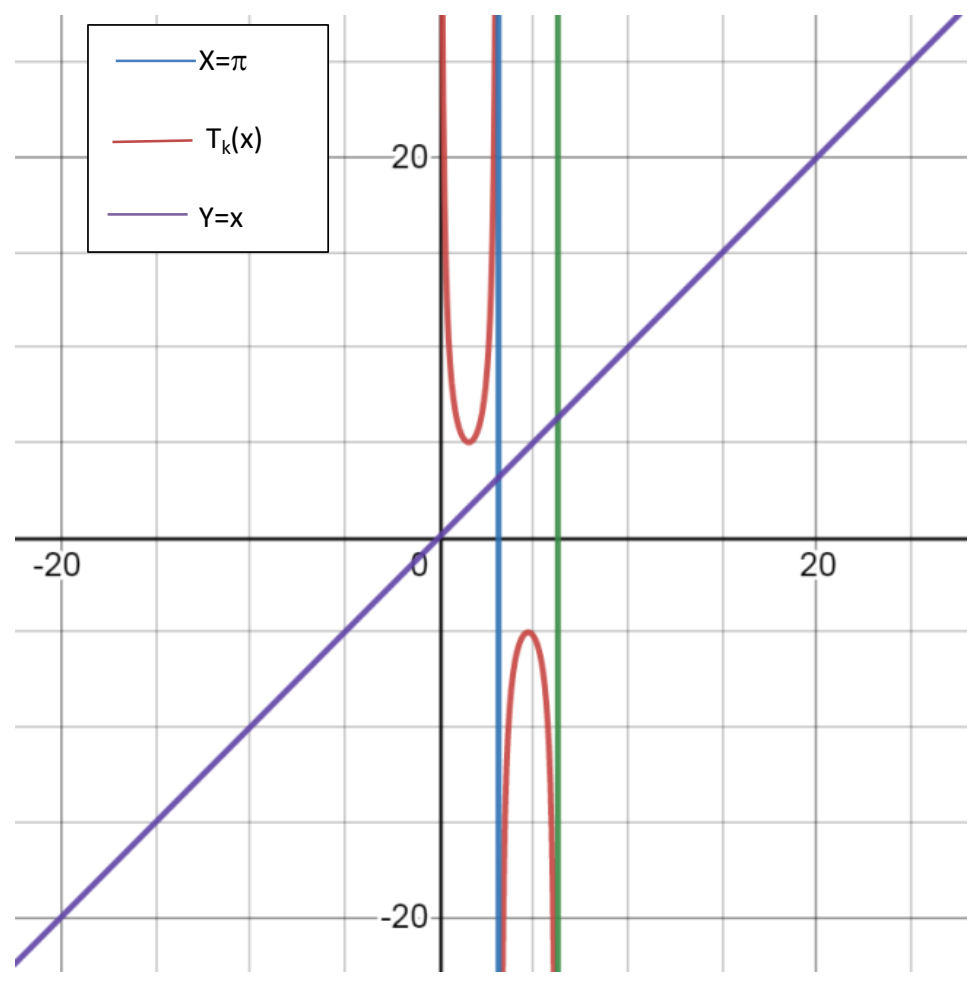

Figure 8- $\left.T_{k}(x)=f_{k}(x)-x, \mathrm{k}>\mathrm{k}_{3}\right)$ 


\section{References}

[1] Devaney, R.L., "Dynamics Topology, and Bifurcations of Complex exponentials", Topology Appl., vol. 110, pp. 133-161, 2001.

[2] Devaney, R.L., "A survey of exponential dynamics ", Chapman and Hall/CRC, pp. 105-122, 2004.

[3] Al-Husseiny, H., F.," A Study of the Dynamics of the family $\lambda \frac{\sinh ^{m} z}{z^{2 m}}$ ", Iraqi Journal of Science, vol. 4, no. 52, pp. 494-503, 2011.

[4] Sajid, M., "Real and Complex Dynamics of One Parameter Family of Meromorphic Functions", Far East .Dyn. Syst., vol. 19, no. 2, pp. 89-105, 2012.

[5] Akbari, M., Rabii, M., "Real Cubic Polynomials With a Fixed Point of Multiplicity Two", Indagationes Mathematicate, vol. 26, pp. 64-74, 2015.

[6] Magrenan, A., Gutierrez, J., "Real Dynamics for Damped Newtons Method Applied to Cubic Polynomials", Comput. Appl. Math., vol. 275, pp. 527-538, 2015.

[7] Radwan, A. G., "On Some Generalized Discrete Logistic Maps", J. Adv. Res., vol. 4, no. 2, pp. 163-171, 2013.

[8] Faris, S. M., "Dynamics of Certain Families of Transcendental Meromorphic Functions", Ph.D. thesis University of Baghdad, 2006.

[9] Jamil, Z. Z. and Hussein, Z., "Common Fixed Point of Jungck Picard Itrative for Two Weakly Compatible Self-Mappings", Iraqi Journal of Science, vol. 62, no. 5, pp. 1695-1701, 2021.

[10] Sajid, M., "Singular Values and Real Fixed Points of One-Parameter Families Assogiated with Fundamental Trigonometric Functions sinz, $\cos \mathrm{z}$ and $\tan \mathrm{z}$ ", International Journal of Applied Mathematics, vol. 33, pp. 635-647, 2020. 